Cahiers $d u$ MONDE RUSSE

\section{Cahiers du monde russe}

Russie - Empire russe - Union soviétique et États indépendants

$48 / 4 \mid 2007$

Varia

\title{
Svetlana Tabatchnikova, Le Cercle de méthodologie de Moscou (1954-1989)
}

\section{Daniela Steila}

\section{OpenEdition \\ Journals}

Édition électronique

URL : https://journals.openedition.org/monderusse/6098

DOI : 10.4000/monderusse.6098

ISSN : $1777-5388$

Éditeur

Éditions de l'EHESS

\section{Édition imprimée}

Date de publication : 2 décembre 2007

Pagination : 764-766

ISBN : 978-2-7132-2148-4

ISSN : $1252-6576$

Référence électronique

Daniela Steila, "Svetlana Tabatchnikova, Le Cercle de méthodologie de Moscou (1954-1989)», Cahiers du monde russe [En ligne], 48/4 | 2007, mis en ligne le 16 juin 2009, consulté le 02 septembre 2022. URL : http://journals.openedition.org/monderusse/6098 ; DOl : https://doi.org/10.4000/ monderusse.6098

Ce document a été généré automatiquement le 2 septembre 2022.

Tous droits réservés 


\title{
Svetlana Tabatchnikova, Le Cercle de méthodologie de Moscou (1954-1989)
}

\author{
Daniela Steila
}

\section{RÉFÉRENCE}

Svetlana Tаватснniкоva, Le Cercle de méthodologie de Moscou (1954-1989). Une

pensée, une pratique. Paris : École des hautes études en sciences sociales, 2007, 332 p.

1 Svetlana Tabatchnikova se penche sur un phénomène philosophique, pédagogique, politique et culturel très original : le Cercle de méthodologie de Moscou (CMM). Elle en explore ici tantôt les vicissitudes historiques, tantôt les formulations théoriques sur fond d'histoire culturelle et politique de la Russie, de la mort de Stalin à la chute de l'URSS. Ce Cercle est, pour la première fois, examiné à partir de sources multiples et d'analyses approfondies. Il a pendant des décennies impliqué dans ses activités de nombreux intellectuels issus de formations différentes, qui y reçurent un véritable apprentissage de pensée critique. Cette expérience fait partie de ce domaine intermédiaire entre orthodoxie marxiste du parti et véritable dissidence, dont l'impact et le sens dans l'histoire culturelle soviétique forment le fond de ce travail. S. Tabatchnikova part de sources hétérogènes (articles, interventions au cours de rencontres, conférences et séminaires, documents d'archives publics et privés, entretiens avec des protagonistes et des témoins...), convoquées avec une imposante assurance méthodologique. Elle détermineainsiun cadre à différents niveaux : l'histoire du CMM s'y conjugue avec les événements de la transformation du système de pouvoir, des structures de transmission de la connaissance, l'« expérience » enfin de vie et de pensée des intellectuels soviétiques à l'intérieur de ce système.

2 Ainsi la rencontre, au début des années 1950, d'Aleksandr Zinov'ev, de Boris Grušin, de Merab Mamardašvili et de Georgij Ščedrovickij, les quatre protagonistes du premier 
« cercle» philosophique, est réévaluée sur deux plans. D'une part sur le fond de la "catastrophe anthropologique» des décennies précédentes (l'expression est de Mamardašvili), due à la négation systématique de la dignité individuelle pratiquée par le pouvoir et à laquelle les individus réagissaient par des attitudes différentes (adaptation sans réserves, doutes sur leur capacité à comprendre, sentiment de rupture totale avec la réalité, et autres stratégies de survie); d'autre part, sur la situation institutionnelle ou générationnelle de l'enseignement philosophique. Apparaît alors, encore à l'époque stalinienne, un intérêt spécial pour la logique. Le premier noyau du futur Cercle se constitue à l'Institut de philosophie en étudiant la logique du Capital de Marx, en particulier le problème du rapport abstrait/concret. C'est l'objet à l'époque de recherches d'un autre groupe, lui aussi « honnêtement critique » envers les institutions, réuni autour d'Evald Il'enkov. Alors que ce dernier adoptait une position qu'on pourrait qualifier de néohégélienne, le groupe de Zinov'ev travaillait sur la négation de l'identité de l'être et de la pensée. Ces questions de logique furent débattues officiellement, après la mort de Stalin, en deux séminaires à l'Institut de philosophie au cours du premier semestre de 1954. Les quatre amis y poursuivent pour la première fois une stratégie commune d'interventions publiques qui sera une de leurs caractéristiques. L'espace public de discussion se ferme cependant déjà en 1956 et l'année suivante les quatre protagonistes se séparent. S. Tabatchnikova voit à la base de cette rupture l'intention de Ščedrovickij d'élaborer une nouvelle logique comme "théorie de la pensée ». Or Zinov'ev maintenait sa réflexion dans le cadre de la logique formelle traditionnelle. Entrent également en compte des divergences sur les stratégies communes d'intervention publique.

3 L'âme du Cercle méthodologique, qui surgit de cette première expérience, est Georgij Ščedrovickij, penseur rigoureux, d'une grande dignité personnelle. Autour de lui se rassemble un séminaire "restreint ", généralement dans des maisons privées, et un séminaire plus ouvert (jusqu'à plusieurs dizaines de participants), sous des « couvertures » institutionnelles variables, selon ses pérégrinations, d'une institution, plus ou moins marginale, à l'autre.

4 S. Tabatchnikova se propose de périodiser l'activité du Cercle en trois époques, selon les évolutions des problématiques envisagées et des stratégies adoptées. Dans la première période, de la formation à la fin des années 1950, on élabore une théorie de la pensée en tant qu'activité considérée dans la continuité entre la formation d'une connaissance et son application pratique. Repoussant l'identité de l'être et de la pensée, de la forme et du contenu de la connaissance, le Cercle parvient à une conception de l'objet en tant que corrélé à l'activité humaine, théorique et pratique. Le processus de la pensée, reconstitué à partir des contributions interdisciplinaires des membres du Cercle, est un «modèle configurateur». La description du processus de la pensée prend une valeur de prescription: c'est proprement une méthode.

5 La deuxième période comprend les deux décennies suivantes. Le Cercle se dédie alors à la théorie de l'activité. Quelle élaboration conceptuelle adéquate pour rendre compte de l'activité dans des situations concrètes? Les contributions de la linguistique, de la psychologie, de la sociologie, mais aussi de l'ingénierie et du management sont convoquées. L'activité est entendue ici comme un «système» impersonnel, dont la cohérence dépend du processus de sa reproduction, articulé entre le niveau normatif et la réalisation concrète, articulation où s'insère l'action responsable de l'individu. Dans cette perspective, l'intérêt du Cercle se déplace : « On ne cherche plus de modèle pour la 
théorie de la pensée, mais on étudie l'organisation de la science, ses structures internes et externes » (p. 148). De là découle une réflexion approfondie et novatrice sur le concept de "système", examiné dans ses aspects fonctionnels, morphologiques, matériels, dynamiques; et c'est là aussi que le Cercle se heurte à " une antinomie entre les processus de la pensée et de la réflexion, source du développement, et les processus globaux de l'activité, celle-ci apparaissant comme un principe de fixation, de reproduction et de fonctionnement normatif » (p. 155).

6 La réarticulation des concepts de pensée et d'activité est proposée à partir de la fin des années 1970, dans la troisième phase de l'activité du Cercle, à l'époque de la "stagnation ", alors que la situation professionnelle de ščedrovickij s'aggrave : exclu du parti en 1968, il trouve refuge au Laboratoire de méthodes expérimentales pour l'enseignement qui dépend de l'Union des peintres de l'URSS. Parmi les membres du Cercle et dans la société prévaut un terrible sentiment d'impuissance, tant la situation politique et culturelle semble immuable. Dans ce climat, Ščedrovickij insiste pour déterminer de nouvelles perspectives de travail, tant sur le plan conceptuel, avec l'élaboration du nouveau concept de « pensée-action », que sur le plan pratique, avec la proposition des premières expériences de «jeu d'organisation». Il s'agit d'une expérience (à laquelle $\mathrm{S}$. Tabatchnikova a elle-même participé) qui exige une grande implication. Un nombre parfois très élevé de personnes qui partagent un même problème concret à résoudre s'engagent pendant des jours à analyser la situation, à définir les objectifs particuliers et communs, à réfléchir sur les conflits et les dynamiques qui se présentent... Tout cela provoque une problématisation et une déconstruction des structures disciplinaires rigides et des expériences personnelles intenses: mise en question de soi, partage des points de vue, prise de responsabilités. S. Tabatchnikova observe : «À l'époque où l'idéologie des gouvernés, en réaction à l'idéologie restrictive et pesante des gouvernants, consistait à croire que l'on ne pouvait rien faire du tout [...], le jeu offrait à chacun une possibilité pour comprendre la situation et pour agir, et invitait à se constituer en acteur responsable » (p. 191).

7 Après avoir rapidement tracé la multiplication de ce genre d'expériences, souvent conduites par les membres du CMM pendant les années tumultueuses de la perestroïka, S. Tabatchnikova conclut sur la mort du leader incontesté. Ščedrovickij meurt en 1994 à 65 ans, regretté par tous ceux qui l'ont connu et qui continuent à le considérer comme un « maître ».

8 S. Tabatchnikova est consciente que certains thèmes et réflexions du CMM peuvent sembler familiers au lecteur occidental (épistémologie scientifique de Piaget, cybernétique, conception de l'objet chez Husserl, dynamique de groupe de Lewin...), mais elle envisage surtout les rapports et les influences propres au contexte de l'époque. D'une manière cohérente, le dernier chapitre replace l'expérience du CMM dans le cadre des spécificités de la pensée philosophique russe en général et du rôle de l'intelligentsia à l'égard duquel le Cercle marque une rupture. Le Cercle n'apparaît pas seulement comme un lieu de réflexion originale et "critique», mais aussi comme un laboratoire de "sociabilité alternative»: "le jeu d'organisation met au centre un espace de communication et de construction de sens [...] où l'individu et le groupe, la volonté et la raison, la critique et l'argumentation doivent s'articuler pour rendre possible un projet commun » (p. 206). L'auteur voit ici un riche héritage à peine exploité.

9 Un précieux appendice de documents de Ščedrovickij clôt le volume: des notes autobiographiques complétées par d'autres témoignages, un essai qui donne un 
échantillon de son style philosophique; ses souvenirs et ceux de Mamardašvili sur les origines du Cercle. Les notices biographiques des principaux personnages enfin contribuent à faire de ce livre une référence indispensable pour l'histoire culturelle soviétique de la deuxième moitié $\mathrm{du} \mathrm{xx}^{\mathrm{e}}$ siècle. 